\title{
THE OPTICAL FUTURE OF HIGH-SPEED NETWORKS
}

\author{
C. Qiao \\ State University of New York, Buffallo
}

\begin{abstract}
:
With the ever increasing optical transmission rate, now exceeding $1 \mathrm{~Tb} / \mathrm{s}$ on a single fiber thanks to Wavelength Division Multiplexing (WDM) technologies, electronic routing/switching is quickly becoming a performance bottleneck in high-speed backbone networks. WDM optical networking, though rooting in the physical and link layers, is by no means yet another ordinary low layer technology as it may affect the designs of the upper electronic layer. Specifically, WDM optical networks can be configured to bypass intermediate electronic components by switching/routing data in the optical domain. This will not only reduce electronic processing and $\mathrm{I} / \mathrm{O}$ loads, but also provide bit-rate and coding format transparency. However, this may also lead to unexpected "shortest" and "alternate" paths in the electronic layer. In addition, primitive optical logic, and especially the lack of optical memory (buffer) are major challenges in order to realize the vision of building a bandwidth-abundant infrastructure, which is ubiquitous and yet efficient, based on WDM optical networks. In this talk, I will describe these issues and along relevant optical switching paradigms, namely wavelength-routing (as a form of optical circuit switching), optical packet switching, and optical burst switching (OBS), and discuss how the next generation Optical Internet may support QoS and provide multicast services in the WDM optical layer.
\end{abstract}

The original version of this chapter was revised: The copyright line was incorrect. This has been corrected. The Erratum to this chapter is available at DOI: 10.1007/978-0-387-35580-1_16 
Chunming Qiao earned his BS degree in Computer Science and Engineering from University of Science and Technology of China (USTC) in Hefei, P.R. China. He received the Andrew-Mellon Distinguished doctoral fellowship award from University of Pittsburgh and later earned his Ph.D. degree in Computer Science there in 1993. He was an Assistant Professor in the ECE Department, and now an Associate Professor in the newly created CSE Department at SUNY at Buffalo. His research interests cover the two converging areas of computers and communications and currently, he is conducting research on Wavelength Division Multiplexed (WDM) networks and internetworks (e.g. IP over WDM) which has been funded by NSF and Telcordia Technologies (formerly Bellcore). Dr. Qiao has published more than 50 papers in IEEE Trans. on Computers, Trans on Parallel and Distributed Systems, Trans. on Communications and Trans. on Networking as well as other journals and conference proceedings. He has served as a CoChair for the annual All-optical Networking conference since 1997, a Program Vice Co-Chair for the 1998 International Conf. on Computer Communications and Networks (IC3N), panel/session organizer at the IEEE Globecom'99 and IEEE MILCOM'96, and program committee members and session chairs in over a dozen of other conferences and workshops. He has been featured in a couple of interviews shown in the Alcatel's Communications Week and SPIE's OE Reports. He is also an editor of the Journal on High-Speed Networks (JHSN) and the new Optical Networks Magazine, and a member of IEEE Computer Society and IEEE Communications Society. 